\title{
Ocorrência da espécie invasora Corbicula fluminea (Bivalvia, Corbiculidae) no rio Sapucaí (São Paulo, Brasil)
}

\author{
Marina Peixoto Vianna* \\ Wagner Eustáquio Paiva Avelar \\ Laboratório de Malacologia, Departamento de Biologia \\ Faculdade de Filosofia, Ciências e Letras de Ribeirão Preto \\ Universidade de São Paulo - USP \\ CEP 14040-901, Ribeirão Preto - SP, Brasil \\ *Autor para correspondência \\ mpvianna@usp.br
}

Submetido em 06/11/2009

Aceito para publicação em 28/04/2010

\section{Resumo}

As invasões biológicas estão adquirindo importância cada vez maior, tanto pelos problemas ecológicos causados quanto pelos impactos econômicos. Corbicula fluminea (Müller, 1774), é um bivalve exótico invasor introduzido no Brasil na década de 1970, que colonizou os principais rios das regiões sul e sudeste do país. A espécie, devido às adaptações ao ambiente, à velocidade de colonização e ao sucesso reprodutivo, entre outros parâmetros, ocupa rapidamente os substratos preferenciais da fauna de bivalves nativos. O presente estudo registra a ocorrência da espécie invasora no rio Sapucaí, estado de São Paulo. Foram realizadas amostragens em dois pontos com sedimentos diferentes, arenoso e lodoso. Foram realizadas análises granulométricas e determinado o teor de matéria orgânica dos sedimentos. Os animais encontrados em substrato arenoso apresentaram maiores densidades, maiores médias de tamanho e, consequentemente, maiores médias de biomassa.

Unitermos: bivalve, Corbicula fluminea, molusco, ocorrência

\section{Abstract}

Occurrence of the invasive species Corbicula fluminea (Bivalvia, Corbiculidae) in the Sapucaí River (São Paulo, Brazil). Biological invasions are gaining increasing importance due to the ecological damage and economic impacts caused by the invaders. Corbicula fluminea (Müller, 1774) is an invasive exotic bivalve introduced into Brazil in the 1970's, and it has colonized the major rivers of the south and southeast. This species, owing to its adaptations to the environment, speed of colonization and reproductive success among others parameters, quickly occupies the substrates preferred by the natives. The present study registers its occurrence in Sapucaí River, São Paulo State. Samples were taken at two different sites of the river with different substrates, one sandy and another muddy. Granulometric analysis and determination of the organic matter content of the sediment were then conducted. The individuals captured in sandy sediment showed higher densities, higher average size, and therefore higher average biomass.

Key words: bivalve, Corbicula fluminea, mollusc, occurrence 


\section{Introdução}

Corbicula fluminea (Müller, 1774) é um molusco bivalve de água doce pertencente à família Corbiculidae, endêmico do sudeste da Ásia (McMahon, 1982).

O primeiro trabalho que identificou e registrou a presença da espécie asiática no continente americano, especificamente nos EUA, foi o de Burch (1944, apud Mansur e Garces, 1988).

A introdução de C. fluminea na América do Sul ocorreu, provavelmente, entre 1965 e 1975, tendo o primeiro registro no estuário do rio da Prata, na Argentina (Ituarte, 1981). Veitenheimer-Mendes (1981) registrou pela primeira vez, a presença de representantes asiáticos do gênero Corbicula no sul do Brasil. A partir daí, inúmeros trabalhos mostraram a ocorrência e dispersão da espécie na região sul do país e, pouco tempo depois, já em regiões mais distantes. Dentre eles, Mansur e Garces (1988) no Rio Grande do Sul, Callil e Mansur (2002) na região do Pantanal brasileiro, ambos mencionados por Mansur et al. (2004) numa retrospectiva e mapeamento da invasão de C. fluminea na América do Sul. Ainda, Beasley et al. (2003) na bacia amazônica, Martins et al. (2004) no Rio Grande do Sul, Rodrigues et al. (2007) no lago Paranoá em Brasília, Suriani et al. (2007) e Pimpão e Martins (2008) em outra região da bacia Amazônica. No estado de São Paulo, Avelar (1999) foi o primeiro a registrar a ocorrência da espécie asiática na bacia do rio Mogi-Guaçu. A partir daí, Vianna (2002) iniciou os estudos sobre biologia de C. fluminea no rio Sapucaí.

O sucesso adaptativo da espécie se deve à sua resistência ao estresse ambiental, tolerância a diferentes substratos, alta capacidade reprodutiva, rápido crescimento e capacidade de filtrar grandes volumes de água (Graney et al., 1980; McMahon, 1982). Assim, C. fluminea se tornou um importante membro de muitas comunidades bentônicas de água doce. Em alguns rios arenosos, esta espécie pode dominar a comunidade de bivalves bênticos numericamente e em termos de biomassa (Hakenkamp e Palmer, 1999).

Diversos autores registraram altas densidades de $C$. fluminea onde a espécie se estabeleceu. Mansur e Garces (1988), em um estudo na Estação Ecológica do Taim, no Rio Grande do Sul, comprovaram a alta densidade de $C$. fluminea, com o máximo de mais de 5.000 indivíduos/ $\mathrm{m}^{2}$, em aproximadamente cinco anos. Também no Rio Grande do Sul, Mansur et al. (1994) verificaram maior abundância da espécie em ambiente lótico do que lêntico, com preferência por áreas marginais onde o declive é suave. Callil e Mansur (2002) encontraram densidades baixas de C. fluminea na região do Pantanal - Brasil (192 indivíduos $/ \mathrm{m}^{2}$ ), o que pode estar relacionado à recente colonização na região. Suriani et al. (2007) encontraram densidade máxima de 5503 indivíduos/ $\mathrm{m}^{2}$ na represa de Bariri-SP. Sousa et al. (2008) relatam densidades variando de 80 a 4185 indivíduos $/ \mathrm{m}^{2}$, ao estudarem populações da espécie invasora em rios de Portugal. Densidades, a partir de 200 indivíduos $/ \mathrm{m}^{2}$, foram consideradas como potencial para causar danos, tanto na canalização de águas, quanto para usinas hidroelétricas (Mansur e Garces, 1988). Isso porque grandes aglomerados desses animais podem obstruir a passagem da água nos sistemas de captação de usinas hidroelétricas e de abastecimento de água.

De acordo com Mansur e Garces (1988), as altas densidades de C. fluminea também causam sérios problemas às populações nativas de bivalves em geral, devido à competição por espaço e nutrientes. Beasley et al (2003), ao registrarem a primeira ocorrência de $C$. fluminea na bacia amazônica, já relatam a dominância da espécie em relação às nativas. Em alguns pontos os bivalves nativos desapareceram e somente a espécie invasora é encontrada. Segundo Lee e DeAngelis (1997), as populações de bivalves límnicos na América do Norte, com grande distribuição ao longo de muitos sistemas hidrográficos, têm mostrado um declínio no seu tamanho com o passar dos anos devido às atividades antropogênicas e, principalmente, à competição com espécies exóticas. Vaughn e Spooner (2006) fizeram uma comparação entre $C$. fluminea e os bivalves nativos, mostrando que a espécie asiática é menor que as nativas, ocorre em grandes aglomerados, e apresenta uma maior taxa de filtração. Essa alta taxa de filtração provavelmente limita a disponibilidade de alimento na coluna d'água para os indivíduos adultos.

O objetivo do presente estudo é registrar a ocorrência da espécie invasora Corbicula fluminea no rio Sapucaí$\mathrm{SP}$, em pontos com características sedimentológicas 
distintas. Foram comparadas densidades, morfometria e biomassa das populações em três períodos diferentes. Os dados obtidos neste estudo contribuirão para o conhecimento acerca da biologia da espécie invasora, ainda pouco estudada, nos mananciais do Estado de São Paulo.

\section{Material e Métodos}

O rio Sapucaí, importante afluente do rio Grande, faz parte da bacia hidrográfica Sapucaí/Grande, com área de drenagem de $9.121 \mathrm{~km}^{2}$ e vazão média de $146 \mathrm{~m}^{3} / \mathrm{s}$ (Figura 1). Suas águas são usadas no abastecimento público e industrial, afastamento de efluentes domésticos e industriais, da rede para o ponto de descarga ou tratamento através de emissários e, irrigação de hortaliças e plantas frutíferas (CETESB, 2009).

Duas áreas distintas foram delimitadas no rio, georreferenciadas com auxílio de um GPS: uma com substrato de composição lodosa (20³0'57,3"S e $\left.47^{\circ} 50^{\prime} 14,5^{\prime \prime} \mathrm{W}\right)$ e outra com substrato arenoso $\left(20^{\circ} 30^{\prime} 52,6^{\prime} \mathrm{S}\right.$ e $\left.47^{\circ} 50^{\prime} 20,9^{\prime} \mathrm{W}\right)$ para verificar diferenças na densidade populacional, morfometria, biomassa das populações. As áreas escolhidas distam, aproximadamente, $250 \mathrm{~m}$ (em linha reta) entre si, com um trecho de corredeira entre elas.

Foram realizadas três coletas nos pontos estabelecidos: início do período das chuvas (outubro/2001), quando o nível dos rios começa a aumentar; época de cheia dos rios (dezembro/2001) e época de seca (junho/2002), quando o nível rio atinge seu mínimo.

\section{Estudos populacionais}

Os animais foram localizados tateando-se o fundo do leito do rio até aproximadamente $1 \mathrm{~m}$ de profundidade. Foi utilizado um quadrado feito de PVC (dimensões de $1 \mathrm{~m} \mathrm{X} \mathrm{1m),} \mathrm{para} \mathrm{delimitar} \mathrm{o} \mathrm{espaço} \mathrm{a} \mathrm{ser} \mathrm{coletado}$ no fundo do rio, com três réplicas em cada ponto. Os quadrados foram dispostos próximos entre si, sobre os bancos de $C$. fluminea. Todos os animais presentes no espaço do quadrado foram retirados manualmente e com o auxílio de peneiras de malha fina $(1 \mathrm{~mm})$ para a coleta do indivíduos jovens.

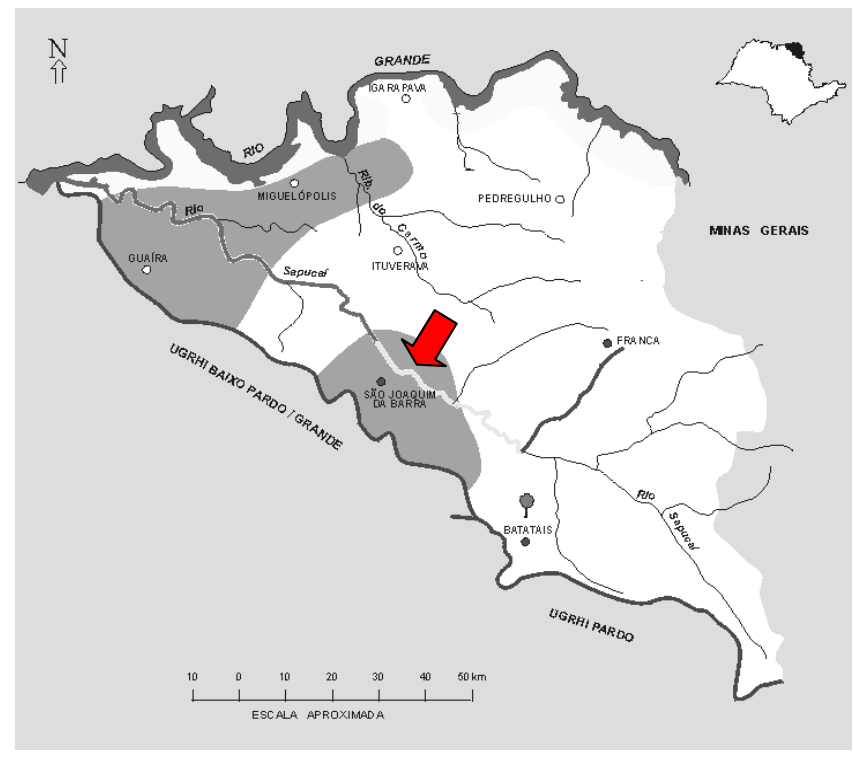

FIGURA 1: Mapa da bacia hidrográfica do rio Sapucaí com a localização da região onde estão as duas áreas de coleta (CETESB, 2009).

Em cada quadrado analisado, amostras do sedimento de fundo foram coletadas, para análise granulométrica e a determinação do teor de matéria orgânica. Tanto os animais como o sedimento, foram acondicionados em sacos plásticos devidamente etiquetados e transportados para o laboratório em caixas térmicas com gelo.

Em laboratório, todos os animais capturados de cada quadrado foram descongelados à temperatura ambiente para os estudos populacionais. Com o auxílio de um paquímetro (precisão $0,01 \mathrm{~cm}$ ), foram tomadas as seguintes medidas: comprimento, largura e altura das conchas, de todos os indivíduos capturados, de acordo com Mansur et al. (1987). Realizadas as medidas morfométricas, as conchas foram abertas e, com o auxílio de um bisturi, as partes moles foram retiradas e pesadas (peso úmido), utilizando-se uma balança de precisão $0,001 \mathrm{~g}$ (Gehaka BG 400). As partes moles de cada animal, bem como as suas conchas, foram então colocadas, separadamente, em grades de metal e levadas à estufa a $60^{\circ} \mathrm{C}$, e diariamente pesadas até obtenção do peso constante. Este período estendeu-se por cinco dias, para se determinar o peso seco das partes moles e o peso da concha dos indivíduos.

A densidade populacional foi estimada em indivíduos $/ \mathrm{m}^{2}$ e a biomassa expressa em $\mathrm{g}$ (peso seco)/ $\mathrm{m}^{2}$. Os coeficientes de correlação entre os pares de 
variáveis morfométricas (comprimento, largura e altura das conchas) também foram determinados.

\section{Sedimentologia}

As porções de sedimento, transportadas em caixas térmicas com gelo e conservadas em congelador, passaram pelo processo de secagem em estufa à $60^{\circ} \mathrm{C}$ até se obter o peso seco. Para isso, as amostras de sedimento foram colocadas para secar, individualmente, em bandejas de alumínio, identificadas uma a uma. O tempo de secagem não foi definido por tratar-se de material coletado em horizonte superficial submerso, tendo uma quantidade de água variável em cada amostra. Sendo assim, as amostras eram pesadas diariamente até que não mais fosse registrada variação no peso.

Após o processo de secagem foi realizado o peneiramento de $50 \mathrm{~g}$ da amostra, separada pelo método de quarteamento (Muehe, 1996). Segundo este método, a amostra é despejada sobre uma superfície, formando um cone. Este cone é dividido em quatro partes iguais, sendo coletado o material de dois quartos opostos. $\mathrm{O}$ procedimento deve ser repetido até a obtenção da amostra no peso desejado. Os $50 \mathrm{~g}$ separados foram peneirados durante $15 \mathrm{~min}$ num agitador de peneiras, comumente usado em análises granulométricas, sendo realizadas três réplicas para cada amostra de sedimento. Esse agitador de peneiras é formado por um conjunto de peneiras de metal com malhas de tamanhos diferentes, com máximo de $2 \mathrm{~mm}$ e mínimo de $125 \mu \mathrm{m}$. O material retido em cada peneira foi pesado em balança eletrônica de precisão $0,001 \mathrm{~g}$, identificando-se o particulado retido segundo a escala de tamanho de sedimentos de Wentworth (1922), com modificações. As frações mais finas do sedimento, silte e argila, não foram separadas.

A quantificação do teor de matéria orgânica, presente no sedimento, foi determinada através da queima de $10 \mathrm{~g}$ de material em mufla a $500^{\circ} \mathrm{C}$, por $3 \mathrm{~h}$, de acordo com Sousa et al. (2008) com modificações. A diferença entre massa inicial e massa final representa a quantidade de matéria orgânica presente na amostra.

\section{Resultados}

A área de sedimento lodoso apresentou, em média, $58 \%$ de silte e argila (as frações mais finas do sedimento) e $42 \%$ das demais frações, com predomínio de areia muito fina. A área de sedimento arenoso apresentou porcentagens de areia muito fina próximas a $80 \%$. A porcentagem de matéria orgânica do sedimento variou de $0,6 \%$ a $8,6 \%$ em sedimento lodoso e de $0,7 \%$ a $1,3 \%$ em sedimento arenoso.

Nas três coletas realizadas no rio Sapucaí registrouse grande variação na densidade de indivíduos das duas populações estudadas de C. Aluminea (Figura 2).

Quando somados jovens e adultos, em sedimento lodoso, a densidade variou de 37 a 956 indivíduos $/ \mathrm{m}^{2}$. Já em sedimento arenoso, a variação registrada foi de zero a 1.282 indivíduos $/ \mathrm{m}^{2}$.
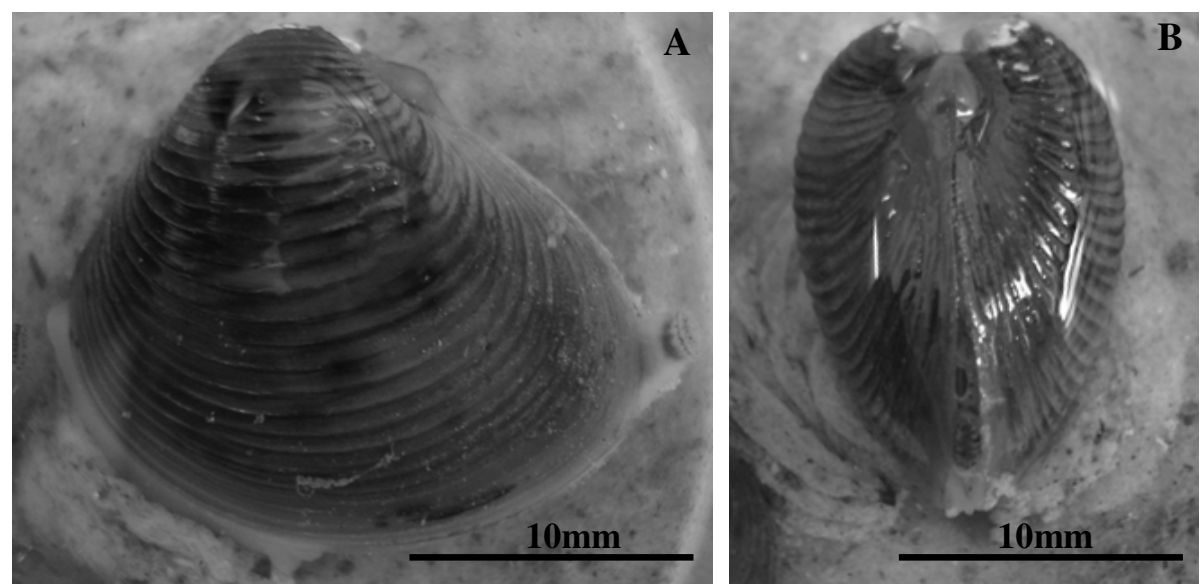

FIGURA 2: Corbicula fluminea. A - Vista externa (lado esquerdo). B - Vista posterior. 
Quando analisados apenas os indivíduos menores que $7 \mathrm{~mm}$, considerados jovens, de acordo com Morton (1977), os resultados foram os seguintes: zero a 51 indivíduos $/ \mathrm{m}^{2}$ em sedimento lodoso e zero a 515 indivíduos $/ \mathrm{m}^{2} \mathrm{em}$ sedimento arenoso. As maiores densidades de jovens foram registradas na coleta de outubro/2001, com destaque para o ponto de sedimento arenoso (Tabela 1). As densidades de indivíduos jovens foram significativamente maiores no ponto de sedimento arenoso $(\mathrm{p}<0,05)$.

TABELA 1: Densidades de indivíduos nas duas áreas amostradas no rio Sapucaí-SP, em três diferentes períodos (adultos: $>7 \mathrm{~mm}$; jovens: $<7 \mathrm{~mm})$.

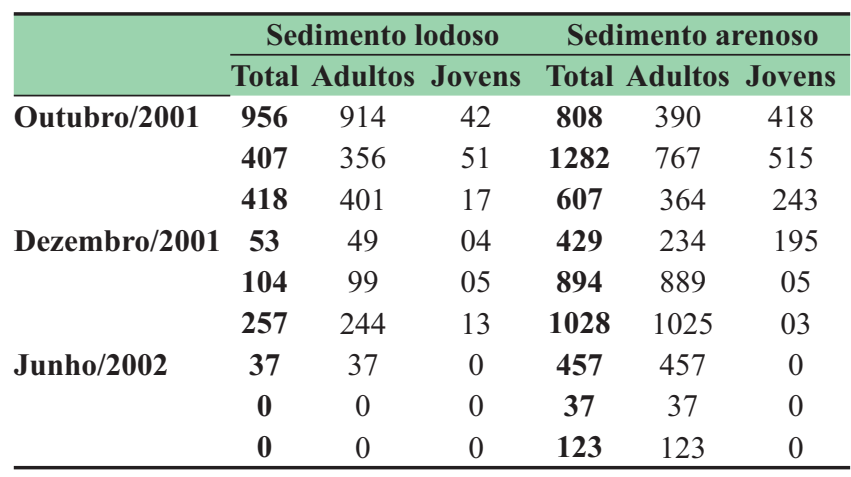

Foram analisados 6.386 animais maiores que $7 \mathrm{~mm}, 2.100$ animais encontrados em sedimento lodoso e 4.286 em substrato arenoso. O tamanho dos indivíduos em sedimento lodoso variou de 13,6 a $23,0 \mathrm{~mm}$ e, em sedimento arenoso, de 11,2 a $26,3 \mathrm{~mm}$. Os maiores exemplares de $C$. fluminea, foram registrados em sedimento arenoso. Mesmo com grande variação na densidade de indivíduos nos dois pontos, não houve diferença significativa entre as medidas de comprimento da concha das duas populações $(p>0,05)$.

Os altos valores obtidos para os coeficientes de correlação (próximos a 1,0) demonstram haver uma estreita relação entre as medidas morfométricas, podendo-se inferir que as populações estudadas desta espécie apresentam crescimento isométrico para estes parâmetros, ou seja, as medidas de comprimento, largura e altura da concha variam nas mesmas proporções. Sendo assim, os resultados asseguram estimativas confiáveis para o cálculo de medidas morfométricas dos indivíduos da população, a partir do registro de um único parâmetro.
Quando comparados os valores de biomassa, observou-se padrão semelhante ao encontrado para as variáveis morfométricas. Os indivíduos encontrados em sedimento arenoso apresentaram valores médios individuais significativamente maiores do que aqueles encontrados em sedimento lodoso $(0,07$ e $0,04 \mathrm{~g}$ de peso seco, respectivamente) $(\mathrm{p}<0,05)$.

É importante destacar que, nas duas primeiras coletas realizadas, não foram encontrados representantes da fauna de bivalves nativos em meio às populações de C. fluminea. Somente na terceira e última coleta, em junho/2002, foram capturados, em sedimento lodoso, exemplares de duas espécies de bivalves nativos: Diplodon rotundus gratus (Wagner, 1827) e Diplodon caipira (Ihering, 1893) (oito e seis exemplares, respectivamente).

\section{Discussão}

Sabe-se que o processo de formação da matéria orgânica depende da flora e da fauna que vive sobre ou dentro do solo. Uma parte considerável da matéria orgânica do solo é formada por raízes e microorganismos (Guerra e Cunha, 1994). As maiores porcentagens de matéria orgânica no sedimento lodoso podem ser resultado da localização da área. O ponto escolhido encontra-se numa região com vegetação próxima à margem e numa área de remanso do rio, o que contribui para o acúmulo de matéria orgânica junto ao sedimento de fundo. O ponto de sedimento arenoso encontra-se numa área com pouca vegetação no entorno e maior velocidade de corrente do rio. De acordo com a classificação de Oliveira (1972), os dois sedimentos podem ser classificados como minerais, pois apresentam porcentagem de matéria orgânica inferior a $20 \%$. Quando os sedimentos apresentam porcentagem de matéria orgânica superior a $20 \%$, estes são denominados sedimentos orgânicos. A diminuição nas porcentagens de matéria orgânica, em relação à primeira coleta, pode estar relacionada ao aumento no volume de água nos meses de novembro, dezembro e janeiro, em decorrência das fortes precipitações que ocorreram no período, consequentemente, aumentando a velocidade de corrente da água. 
A ausência de representantes da fauna nativa, em duas das três amostragens realizadas, vem reforçar a preocupação da comunidade científica com a rápida expansão e colonização de novos habitats pela espécie invasora. Porém, não se pode afirmar que a competição com C. fluminea seja responsável pela falta de representantes nativos nas amostragens no rio Sapucaí, já que o esforço amostral foi pequeno. Pimpão et al. (2008) associam a dificuldade de amostragem e ausência de bivalves nativos às coletas realizadas no período de chuvas, onde o nível dos rios está acima do normal.

A presença de altas densidades de indivíduos jovens (conchas com menos de $7 \mathrm{~mm}$ de comprimento, em média) nas coletas de outubro e dezembro/2001, principalmente em substrato arenoso, indica que esse ambiente permanece em processo de colonização, com um provável pico de recrutamento de $C$. fluminea nos meses que antecederam a coleta realizada (início da primavera).

Vários trabalhos relatam o sucesso reprodutivo de $C$. fluminea e a ocorrência de mais de um pico de liberação de juvenis por ano (Graney et al., 1980; Belanger et al., 1990; Vaughn et al., 2004; Vaughn e Spooner, 2006). Aldridge e McMahon (1978) encontraram dois períodos reprodutivos para $C$. manilensis, sinônimo de $C$. fluminea, um na primavera e outro no outono, com redução ou mesmo ausência reprodutiva, no inverno e meio do verão. Cataldo e Boltovskoy (1999) verificaram um único e bem definido período reprodutivo no ano, estudando populações de $C$. fluminea no delta do rio Paraná, na Argentina. O presente estudo parece corroborar os resultados encontrados para populações da América do Sul, visto que foi possível verificar ao menos um pico reprodutivo, ocorrido antes da amostragem realizada em outubro/2001.

Os maiores valores referentes ao comprimento e biomassa dos indivíduos foram registrados em sedimento arenoso. Segundo Kat (1982), existem inúmeras razões para a redução na taxa de crescimento envolvendo demanda energética e reduzindo a quantidade total de energia disponível para o crescimento. $\mathrm{O}$ autor afirma também, que substratos compostos por partículas muito finas necessitam de um suprimento de energia quase constante para manter a posição do animal no sedimento.
É provável que os animais, quando em substrato lodoso, tenham um gasto energético maior do que aqueles de áreas arenosas para se manterem enterrados no sedimento, diminuindo a energia disponível para outras atividades como crescimento da concha e aumento de peso.

Belanger et al. (1985) puderam verificar, com estudos em laboratório, que $C$. fluminea tem preferência por sedimentos de areia fina e que a densidade da espécie é duas a três vezes maior na areia fina ( $75 \%$ do sedimento com partículas de $0,35-0,60 \mathrm{~mm}$ ) do que em sedimentos de areia grossa ou cascalho. Assim, sugerem que rios com substrato de areia fina e bem oxigenada são os mais adequados para o estabelecimento de populações de Corbicula fluminea, em sítios de colonização recente. Belanger et al. (1990), em rios da América do Norte, verificaram a preferência e abundância da espécie em sedimentos arenosos e com baixa porcentagem de matéria orgânica, onde a média mensal mais alta foi de 839 indivíduos $/ \mathrm{m}^{2}$. Em rios da América do Sul, Cataldo et al. (2001) verificaram a preferência da espécie por sedimentos com alta porcentagem de areia muito fina.

Acredita-se que haja certa dependência da espécie invasora em relação à sedimentologia do local, principalmente para os juvenis. Populações de Corbicula fluminea têm capacidade de colonizar áreas com diferentes tipos de sedimentos, desde seixos e cascalhos até particulado muito fino (silte e argila). Porém é possível que haja uma maior mortalidade de jovens em sedimento muito fino pela obstrução da cavidade palial ou, que esse tipo de sedimento dificulte o assentamento dos jovens na área. As maiores densidades de jovens em sedimento arenoso, no curso médio do rio Sapucaí, corroboram essa hipótese.

Ainda assim, são necessários novos estudos acompanhando o comportamento das populações de $C$. fluminea em um número maior de pontos de estudo e em outras bacias hidrográficas.

\section{Agradecimentos}

Agradecemos a Álvaro da Silva Costa pelo auxílio nos trabalhos de campo e em laboratório. Esse projeto foi financiado pela Fundação de Amparo à Pesquisa do Estado de São Paulo (FAPESP). 


\section{Referências}

Aldridge, D. W.; McMahon, R. F. 1978. Growth, fecundity and bioenergetics in a natural population of the asiatic freshwater clam, Corbicula manilensis Phillipi, from North Central Texas. Journal of Molluscan Studies, 44: 49-70.

Avelar, W. E. P. 1999. Moluscos bivalves. In: Ismael, D.; Valenti, W. C.; Matsumura-Tundisi, T. \& Rocha, O. (Eds). Biodiversidade do Estado de São Paulo, Brasil - Invertebrados de água doce. v.4. Fapesp, São Paulo, Brasil, p.65-68.

Beasley, C. R.; Tagliaro, C. H.; Figueiredo, W. B. 2003. The occurrence of the Asian clam Corbicula fluminea in the Lower Amazon Basin. Acta Amazonica, 33 (2): 317-324.

Belanger, S. E.; Farris, J. L.; Cherry, D. S.; Cairns Jr., J. 1985. Sediment preference of the freshwater Asiatic clam, Corbicula fluminea. The Nautilus, 99 (2-3), 66-72.

Belanger, T. V.; Annis Jr., C. G.; Vanepps, D. D. 1990. Growth rates of the Asiatic clam, Corbicula fluminea, in the upper and middle St. Johns River, Florida. The Nautilus, 104 (1): 4-9.

Callil, C. T.; Mansur, M. C. D. 2002. Corbiculidae in the Pantanal: History of invasion in southeast and central South America and biometrical data. Amazoniana, 17 (1/2): 153-167.

Cataldo, D.; Boltovskoy, D. 1999. Population dynamics of Corbicula fluminea (Bivalvia) in the Parana river delta (Argentina). Hydrobiologia, 380: 153-163.

Cataldo, D.; Colombo, J. C.; Boltovskoy, D.; Bilos, C.; Landoni, P. 2001. Environmental toxicity assessment in the Parana river delta (Argentina): Simultaneous evaluation of selected pollutants and mortality rates of Corbicula fluminea (Bivalvia) early juveniles. Environmental Pollution, 112: 379-398.

CETESB. 2009. Relatório de qualidade das águas interiores do Estado de São Paulo. Companhia de Tecnologia de Saneamento Ambiental, São Paulo, Brasil, 531pp.

Graney, R. L.; Cherry, D. S.; Rodgers Jr., J. H.; Cairns Jr., J. 1980. The influence of thermal discharges and substrate composition of the asiatic clam Corbicula fluminea, in the New River, Virginia. The Nautilus, 94 (4): 130-135.

Guerra, A. J. T.; Cunha, S. B. 1994. Geomorfologia e ambiente. In: Guerra, A. J. T. \&

Cunha, S. B. (Orgs). Geomorfologia: Uma atualização de bases e conceitos. BCD União de Editoras S.A., Rio de Janeiro, Brasil, p.149-209.

Hakenkamp, C. C.; Palmer, M. A. 1999. Introduced bivalves in freshwater ecosystems: The impact of Corbicula on organic matter dynamics in a sandy stream. Oecologia, 119: 445-451.

Ituarte, C. F. 1981. Primeira noticia acerca de la presencia de pelecípodos asiáticos em el área rioplatense. Neotropica, 27: 7982.

Kat, P. W. 1982. Shell dissolution as a significant cause of mortality for Corbicula fluminea (Bivalvia: Corbiculidae) inahbiting acid waters. Malacological Review, 15: 129-134.

Lee, H. L.; DeAngelis, D. L. 1997. A simulation study of the spatio temporal dynamics of the unionids mussels. Ecological Modelling, 95: 171-180.

Mansur, M. C. D.; Callil, C. T.; Cardoso, F. R.; Ibarra, J. A. A. 2004. Uma retrospectiva e mapeamento da invasão de espécies de
Corbicula (Molusca, Bivalvia, Veneroida, Corbiculidae) oriundas do sudeste asiático, na América do Sul. In: Silva, J. S. V. \& Souza, R. C. L. (Eds). Água de lastro e bioinvasão. Ed. Interciências Ltda, Rio de Janeiro, Brasil, p.39-58.

Mansur, M. C. D.; Garces, L. M. M. P. 1988. Ocorrência e densidade de Corbicula fluminea (Muller, 1774) e NeoCorbicula limosa (Maton, 1811) na Estação Ecologia do Taim e áreas adjacentes, Rio Grande do Sul, Brasil (Mollusca, Bivalvia, Corbiculidae). Iheringia, Série Zoológica, 68: 99-115.

Mansur, M. C. D.; Schulz, C.; Garces, L. M. M. P. 1987. Moluscos bivalves de água doce: Identificação dos gêneros do sul e leste do Brasil. Acta Biológica Leopoldencia, 9 (2): 181-202.

Mansur, M. C. D.; Valer, R. M.; Aires, N. C. M. 1994. Distribuição e preferências ambientais dos moluscos bivalves do açude do Parque de Proteção Ambiental COPESUL, município de Triunfo, Rio Grande do Sul, Brasil. Biociências, 2 (1): 27-45.

Martins, D. S.; Veitenheimer-Mendes, I. L.; Faccione-Heuser, M. C. 2004. Corbicula (Bivalvia, Corbiculidae) em simpatria no lago Guaíba, Rio Grande do Sul, Brasil. Biociências, 12 (2): 129-138.

McMahon, R. F. 1982. The occurrence and spread of the introduced Asiatic freshwater clam, Corbicula fluminea (Müller) in North America: 1924-1982. The Nautilus, 96 (4): 134-141.

Morton, B. 1977. The population dynamics of Corbicula fluminea (Bivalvia: Corbiculidae) in Plover Cover Reservoir, Hong Kong. Journal of Zoology London, 181: 21-42.

Muehe, D. 1996. Geomorfologia Costeira. In: Cunha, S. B. \& Guerra, A. J. T. (Eds). Geomorfologia: Exercícios, técnicas e aplicações. Bertrand Brasil, Rio de Janeiro, Brasil, p.191-238.

Oliveira, J. B. 1972. Princípios básicos para classificação e sinopse dos principais grandes grupos de solos do mundo. In: Moniz, A. M. (Coord.). Elementos de Pedologia. Editora Poligono, São Paulo, Brasil, p.357-362.

Pimpão, D. M.; Martins, D. S. 2008. Ocorrência do molusco asiático Corbicula fluminea (Müller, 1774) (Bivalvia, Corbiculidae) no baixo rio Negro, Amazônia Central. Acta Amazonica, 38 (3): 589 592.

Pimpão, D. M.; Rocha, M. S; Fettuccia, D. C. 2008. Freshwater mussel of Catalão, confluence of Solimões and Negro rivers, state of Amazonas, Brazil. Check List, 4 (4): 395-400.

Rodrigues, J. C. A.; Pires-Junior, O. R.; Coutinho, M. F.; MartinsSilva, M. J. 2007. First occurrence of the Asian clam Corbicula fluminea (Bivalvia: Corbiculidae) in the Paranoá Lake, Brasília, Brasil. Brazilian Journal of Biology, 67 (4): 789-790.

Sousa, R.; Rufino, M.; Gaspar, M.; Antunes, C.; Guilhermino, L. 2008. Abiotic impacts on spatial and temporal distribution of Corbicula fluminea (Müller, 1774) in the River Minho Estuary, Portugal. Aquatic Conservation. Marine and freshwater ecosystems, 18: 98-110.

Suriani, A. L.; França, R. S.; Rocha, O. 2007. A malacofauna bentônica das represas do médio rio Tietê (São Paulo, Brasil) e uma avaliação ecológica das espécies exóticas invasoras, Melanoides tuberculata (Müller) e Corbicula fluminea (Müller). Revista Brasileira de Zoologia, 24 (1): 21-32.

Vaughn, C. C.; Gido, K. B.; Spooner, D. E. 2004. Ecosystem processes performed by unionid mussels in stream mesocosm: Species roles and effects of abundance. Hydrobiologia, 527: 3547. 
Vaughn, C. C.; Spooner, D. E. 2006. Scale-dependent associations between native freshwater mussels and invasive Corbicula. Hydrobiologia, 568 (1): 331-339.

Veitenheimer-Mendes, I. L. 1981. Corbicula manilensis (Philippi, 1844) molusco asiático, na bacia do Jacuí e do Guaíba, Rio Grande do Sul, Brasil (Bivalvia, Corbiculidae). Iheringia, Série Zoológica, 60: $63-74$

Vianna, M. P. 2002. Aspectos da biologia de Corbicula fluminea (Müller, 1774) (Corbiculidae) no rio Sapucaí, Estado de São Paulo. Monografia de Graduação, Universidade de São Paulo, Brasil, 51pp.

Wentworth, C. K. 1922. A scale of grade terms for clastic sediments. Journal of Geology, 130: 337-392. 